\title{
Screening genes associated with elevated neutrophil-to-lymphocyte ratio in chronic heart failure
}

\author{
GUOXING WAN $^{1,2}$, LIHUA JI ${ }^{1,2}$, WENBIN XIA ${ }^{1,2}$, LAN CHENG $^{1,2}$ and YONGGANG ZHANG ${ }^{1,2}$ \\ ${ }^{1}$ Department of Cardiology; ${ }^{2}$ Cardiovascular Laboratory, Centre for Translational Medicine, \\ The Second Affiliated Hospital of Shantou University Medical College, Shantou, Guangdong 515041, P.R. China
}

Received January 18, 2018; Accepted May 4, 2018

DOI: $10.3892 / \mathrm{mmr} .2018 .9132$

\begin{abstract}
Neutrophil-to-lymphocyte ratio (NLR) is commonly considered a useful prognostic index for many cardiovascular diseases; however, it has limited sensitivity and specificity. Factors associated with elevated NLR may aid in the prediction of prognosis with heart failure (HF) in combination with NLR. The present study sought to identify decisive factors associated with NLR in HF patients and investigate their association with elevated NLR. The gene expression profile for blood samples from 197 individuals with chronic heart failure $(\mathrm{CHF})$, with corresponding hematological parameters and clinical data were obtained from the public database, GSE77343. Differentially expressed genes (DEGs) were identified, and Gene Ontology and pathway enrichment analyses were performed. The protein-protein interaction network was constructed with the Search Tool for the Retrieval of Interacting Genes along with Cytoscape. Receiver operating characteristic curves for predictive power, sensitivity and specificity were constructed. The present study identified specific associated DEGs by using Pearson linear correlation and logistic regression analysis. A mean NLR of 3.96 was determined as the cutoff value in the analysis. In total, 31 genes were initially identified as DEGs associated with elevated NLR. They were mainly enriched in neutrophil activation and neutrophil mediated immunity, in fluid shear stress and atherosclerosis, and transcriptional misregulation in cancer. Three focused DEGs, solute carrier family 22 member 4 (SLC22A4), interleukin-1 receptor 2 (IL1R2) and vanin 3 (VNN3), were finally revealed to be independently associated with elevated NLR in CHF patients. The present study demonstrated that the three genes SLC22A4, IL1R2 and VNN3 may be independently associated
\end{abstract}

Correspondence to: Professor Yonggang Zhang, Department of Cardiology, The Second Affiliated Hospital of Shantou University Medical College, 69 Dongxia North Road, Shantou, Guangdong 515041, P.R. China

E-mail: zhangyg8686@hotmail.com

Key words: neutrophil to lymphocyte ratio, chronic heart failure, differentially expressed gene with elevated NLR in CHF patients as potential decisive factors of NLR.

\section{Introduction}

Heart failure (HF), commonly the end-stage of most cardiovascular diseases, is a complex inflammatory neuroendocrine syndrome not merely an exclusive problem of low cardiac output $(1,2)$. People older than 65 years with chronic HF (CHF) represent the most with hospitalisation, and approximately $50 \%$ of patients are dead within 5 years of the HF diagnosis $(3,4)$. Despite many advances in diagnosis and treatment approaches, the condition continue to portend a high morbidity and mortality (5). Early identification of patients at high risk and accurate prognostic evaluation is critical for physicians and patients. Although many models of risk stratification and mortality prediction have been developed (6), simple, user-friendly and readily available methods are urgently needed in the clinic. In this regard, the use of hematological factors is becoming increasingly popular as a new approach.

Neutrophil-to-lymphocyte ratio (NLR), a new addition to the list of systemic inflammatory biomarkers, has been repeatedly reported as a prognostic marker and a risk stratification index for various cardiovascular diseases including HF by numerous studies $(5,6)$. Like most commonly used markers for cardiovascular diseases such as C-reactive protein (CRP) and NT-proBNP levels, NLR has become emergingly popular and acceptable as a biomarker for HF in clinical practice (7). NLR has been frequently found to have an significant role in risk-stratification of patients with acute decompensated $\mathrm{HF}$ and a obvious negative prognostic value for long-term mortality by several studies (8-10). Significatively, NLR was evaluated as an inexpensive and readily available marker with similar independent prognostic power as NT-proBNP in elderly patients with CHF (2). Additionally, NLR has also been frequently reported to predict the clinical outcome with other cardiovascular diseases and inflammatory diseases, even malignancies $(5,11)$.

Despite the remarkable significance of NLR in clinical predictability and prognostication, NLR like most inflammatory markers, is less specific to any disease (6), and the mechanism by which elevated NLR is linked to outcomes with HF remains unknown. Therefore, it is rational to propose that the identification of genes coupled with altered NLR 
may contribute to the better understanding of the predictive and prognostic value of NLR in HF and enhance the interpretability of the pathobiology findings. Here, we analyzed the gene signature in blood samples from $197 \mathrm{CHF}$ patients in attempt to screen for potential decisive factors of elevated NLR.

\section{Materials and methods}

Microarrary analysis. The gene expression profile (GSE77343) based on the GPL11532 platform \{(HuGene-1_1-st) Affymetrix Human Gene 1.1 ST Array [transcript (gene)]\} for blood samples from 197 individuals with CHF with corresponding hematological parameters and clinical data were downloaded from the public GEO database (www. ncbi.nlm.gov/geo/), which was deposited by Shannon et al (12). Total RNA isolated from whole blood cells was used to determine the gene expressions. The gene expression data were processed and normalized by using the robust multi-array average method with the $\mathrm{R}$ software (13). Missing data were imputed by the KNN-based method (14). Data were then filtered to include only probe sets with annotations. Differentially expressed genes (DEGs) were identified by significance analysis of the microarrays with the limma package (15), following the criteria 'fold-change $>1.5$ and $\mathrm{P}<0.05$ '. The heat map was plotted for samples and DEGs by using the pheatmap package in $\mathrm{R}$.

Protein-protein interaction (PPI) network, Gene Ontology $(G O)$ and Kyoto Encyclopedia of Genes and Genomes (KEGG) pathway analysis. The online database Search Tool for the Retrieval of Interacting Genes (STRING, string-db.org) was utilized to evaluate the PPI among the DEGs (16). To illustrate the comprehensive information as possible, interactions with a combined score $>0.15$ obtained from STRING were imported into the Cytoscape to construct the PPI network, in which the upregulated DEGs were indicated with red while downregulated genes were indicated with wathet. GO analysis is a commonly used bioinformatics tool for annotating genes by clustering DEGs into three aspects: Cellular component, molecular function, and biological process (17). KEGG is a collection of manually drawn pathway maps representing our knowledge of the molecular interaction and reaction network $f$ or genomes, biological pathways, human diseases, drugs, and chemical substances (18). GO and KEGG pathway analysis were performed with cluster Profiler in $\mathrm{R}(19)$, with $\mathrm{P}<0.05$ as the cut-off.

Statistical analysis. NLR was calculated as neutrophil proportion divided by lymphocyte proportion; the cut-off for NLR was defined as the mean value for all samples at the time of evaluation. NLR greater than the mean value was considered elevated.

Receiver operating characteristic (ROC) curves were utilized to analyze the ability of DEGs in discriminating an elevated NLR and to provide the optimal cut-off point between sensitivity and specificity. The area under the curve (AUC) of ROC was used to evaluate the performance of discrimination. AUC above 0.7 indicated satisfactory diagnostic accuracy, while AUC above 0.8 reflects excellent diagnostic accuracy. To guarantee a high accuracy in the current study, only the
DEGs with an AUC above 0.8 were considered for further analysis. The ROC curves were performed using an R-based online-tool Cutoff Finder (20), which could provide the best cut-off value, the AUC, sensitivity and specificity along with the plot. Additionally, a Pearson linear correlation analysis between NLR and the selected DEGs expression with high diagnostic accuracy was performed with the normalized data.

Based on the cutoff values suggested by the ROC curves, simple logistic regression analyses were performed to further investigate the associations between selected DEGs and NLR adjusted by possible confounders, age and sex. The median age of the cohort was set as the cut-off for the logistic regression. Also, a comprehensive multivariate regression analysis was employed to further identify the significant models including the selected DEGs as well as age and sex. Pearson linear correlation and logistic regression analysis involved use of SPSS 17.0 (SPSS, Inc., Chicago, IL, USA). P<0.05 was considered statistically significant.

\section{Results}

Identification of DEGs. A total of $197 \mathrm{CHF}$ patients with corresponding neutrophil proportion and lymphocyte proportion were included in the study. The mean of NLR was 3.96, which was set as the cut-off for the further analysis. Based on the suggested cut-off for NLR, 31 genes were identified to be differentially expressed with the criteria 'fold-change $>1.5$ and $\mathrm{P}<0.05$, among which 21 genes were upregulated and 10 genes were downregulated in patients with elevated NLR. The heat map for the DEGs was illustrated as Fig. 1A.

PPI network, GO and KEGG pathway analysis. A basic PPI network between DEGs was constructed by using the STRING database, with potential links displayed by Cytoscape (Fig. 1B). To gain the cellular function, process and signal pathway associated with elevated NLR, GO and KEGG pathway enrichment analyses were performed using cluster Profiler. As shown in Fig. 2, the results of GO analysis showed that the DEGs were mainly enriched in neutrophil activation involved in immune response, neutrophil degranulation, neutrophil activation, granulocyte activation and neutrophil mediated immunity. The KEGG pathway analysis revealed that the DEGs were significantly enriched in fluid shear stress and atherosclerosis, transcriptional misregulation in cancer and amoebiasis. These significantly enriched GO terms and pathways will provide further insight into the relation of decisive factors to the elevated NLR in CHF patients for future researches, thereby potentially proposing promising biomarkers for the prognostication and rational interpretability of pathobiology for HF.

ROC curve and Pearson correlation analysis. Six DEGs with an AUC above 0.8 were selected to be potentially related to elevated NLR by the ROC curve analysis. As shown in Fig. 3, ROC curve demonstrated the predicting power with the optimal cut-off on elevated NLR with an AUC value of 0.84 , sensitivity $81.9 \%$, specificity $78.4 \%$ for interleukin-1 receptor 2 (IL1R2), an AUC value of 0.85, sensitivity $80.6 \%$, specificity $79.2 \%$ for solute carrier family 22 member 4 (SLC22A4), an AUC value of 0.82, sensitivity $72.2 \%$, specificity $80 \%$ for vanin 3 (VNN3), an AUC value 
A

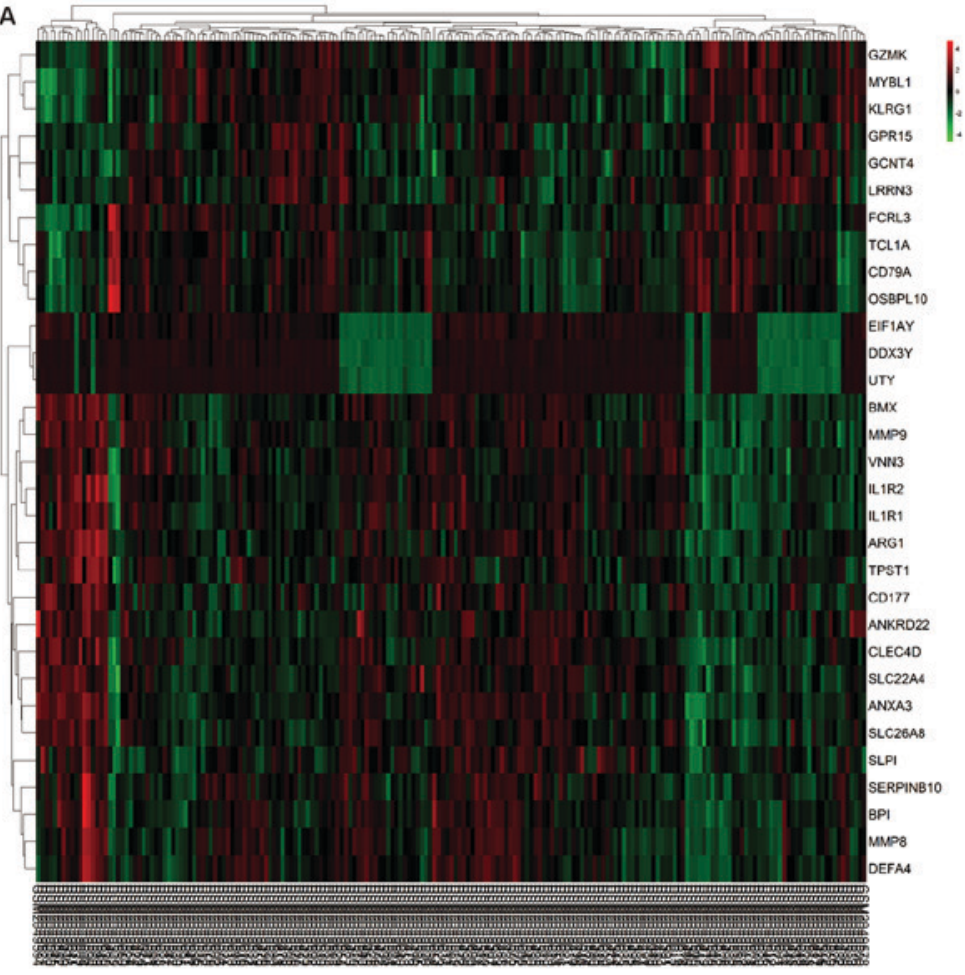

Cluster heatmap for DEGs
B

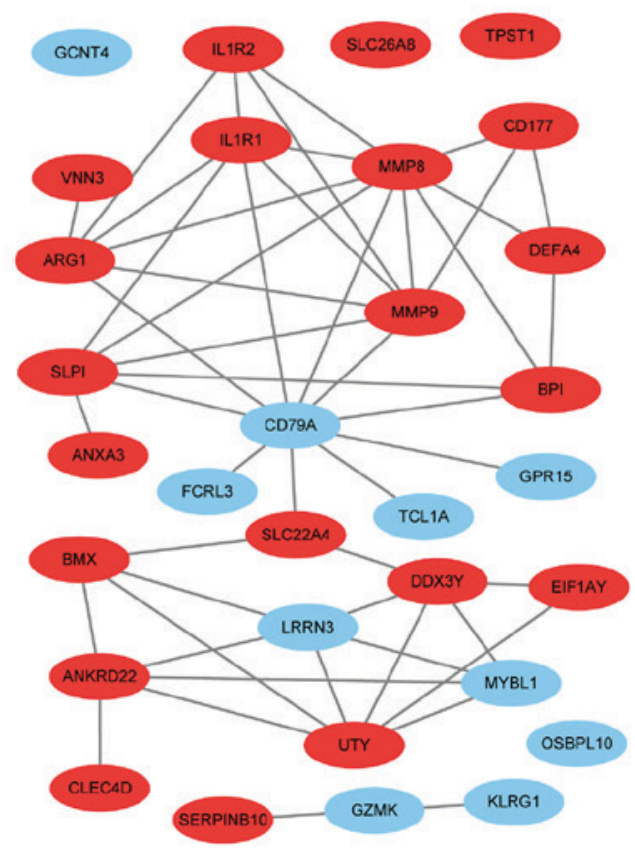

Protein-protein interactions for DEGs

Figure 1. (A) Heatmap and (B) protein-protein interactions for DEGs. DEGs, differentially expressed genes.
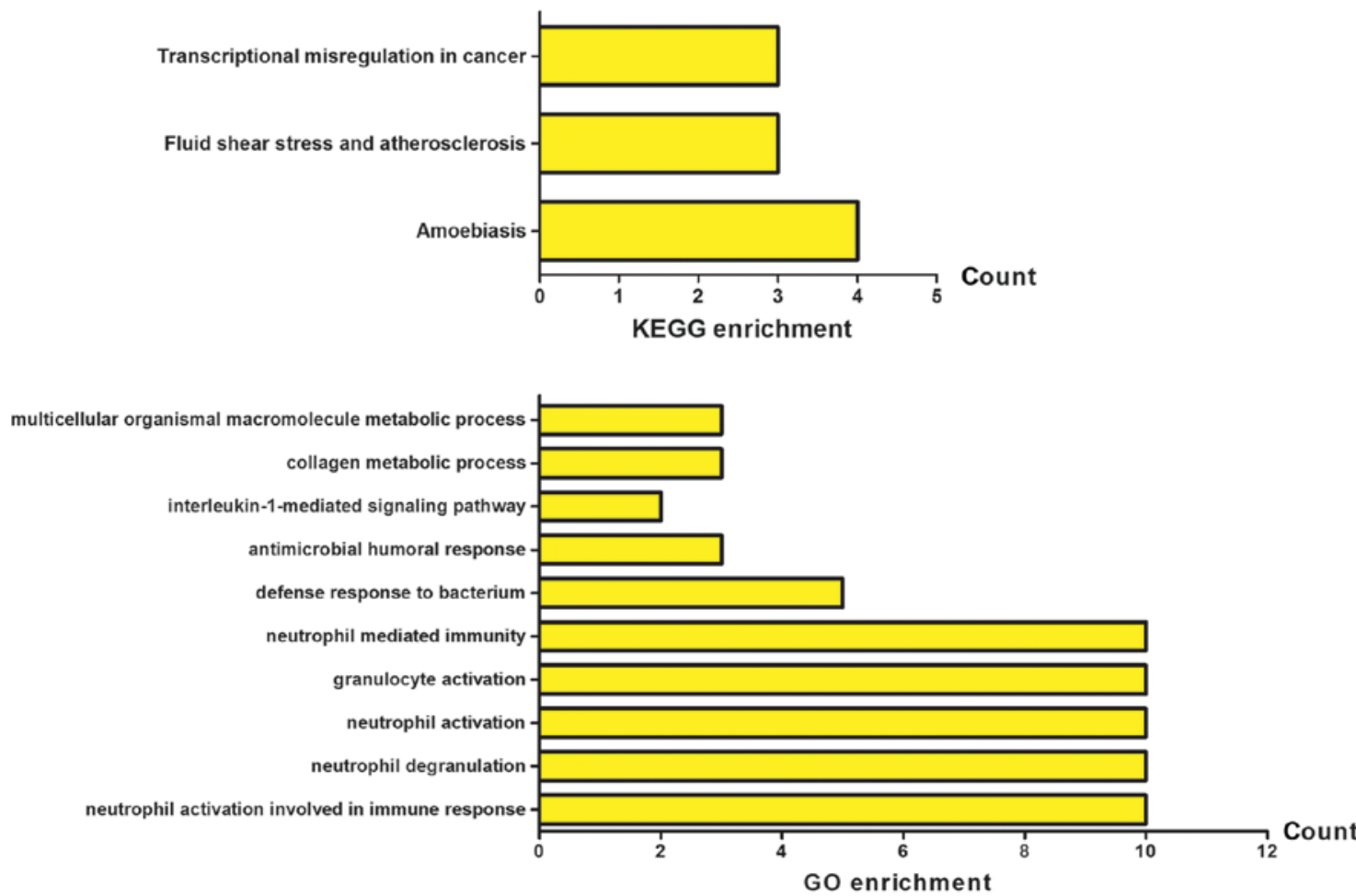

Figure 2. GO and KEGG pathway enrichment analysis. GO, Gene Ontology; KEGG, Kyoto Encyclopedia of Genes and Genomes.

of 0.8 , sensitivity $76.4 \%$, specificity $75.2 \%$ for Annexin A3 (ANXA3), an AUC value of 0.81 , sensitivity $70.8 \%$, specificity $82.4 \%$ for BMX non-receptor tyrosine kinase (BMX) and an AUC value of 0.8 , sensitivity $66.7 \%$, specificity $80.8 \%$ for interleukin-1 receptor type 1 (IL1R1), respectively. To further study the relationships between selected DEGs and NLR, the Pearson relation analysis was performed using the normalized expression data, and the results revealed significant associations of all six genes $(r=0.512, \mathrm{P}<0.001$ for SLC22A4; $r=0.557, \mathrm{P}<0.001$ for IL1R2; $r=0.451, \mathrm{P}<0.001$ 

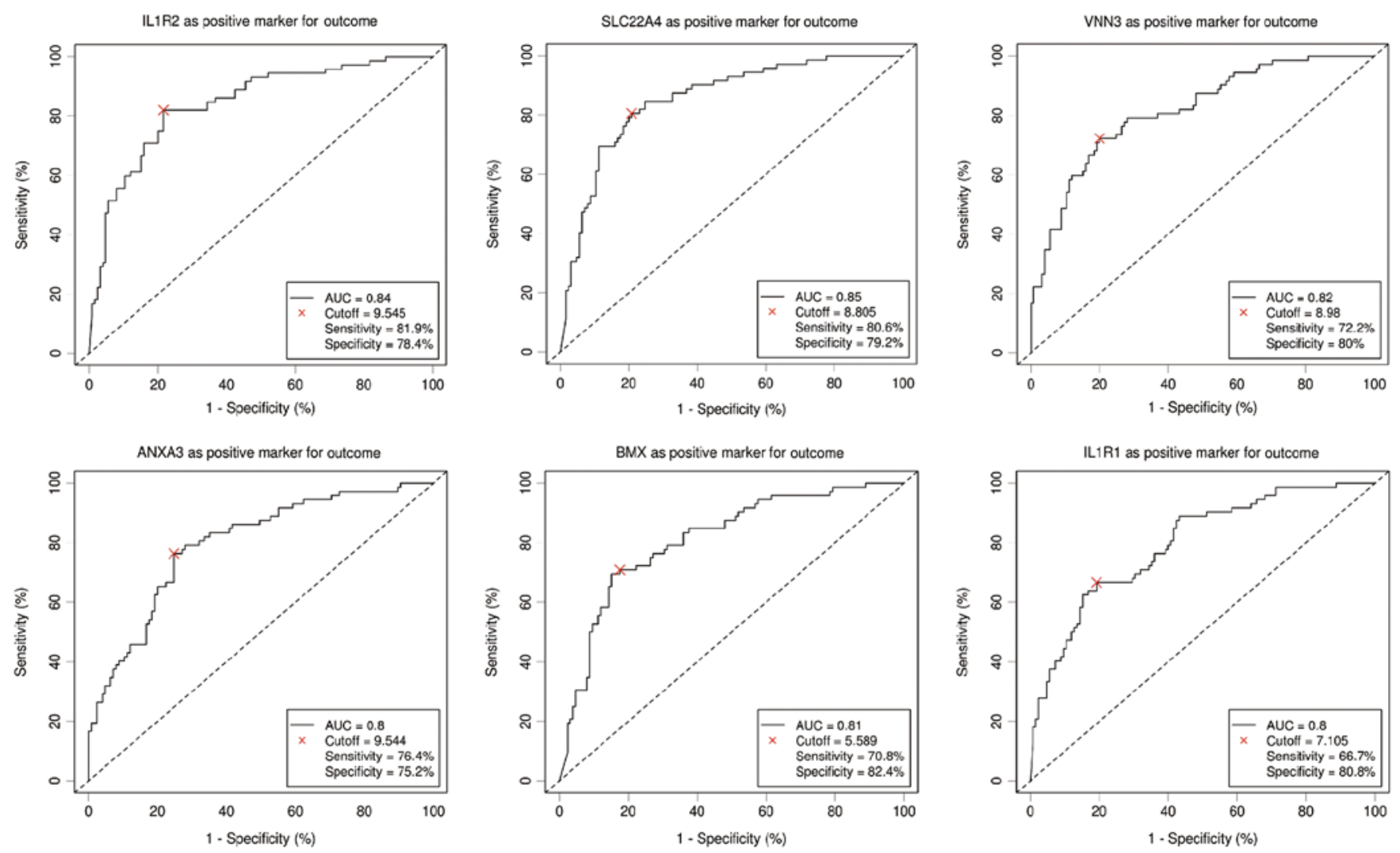

Figure 3. Receiver operating characteristic curves for the six selected genes with an AUC $>0.8$. AUC, area under the curve; SLC22A4, solute carrier family 22 member 4; IL1R, interleukin-1 receptor; VNN3, vanin 3; BMX, BMX non-receptor tyrosine kinase; ANXA3, Annexin A3.

for VNN3; $\mathrm{r}=0.487, \mathrm{P}<0.001$ for $\mathrm{BMX}$; $\mathrm{r}=0.494, \mathrm{P}<0.001$ for ANXA3; $\mathrm{r}=0.470, \mathrm{P}<0.001$ for IL1R1) with elevated NLR (Fig. 4).

Logistic regression analyses. To further study the association of the selected six DEGs with the alteration of NLR, a simple logistic regression adjusted for age and gender was performed. As shown in Table I, significant associations of elevated NLR with SLC22A4, IL1R2, VNN3, ANXA3, BMX, IL1R1 and gender were revealed (all $\mathrm{P}<0.05$ ). Considering the potential links between the DEGs proposed by the PPI network, a multivariate regression analysis including the six DEGs, age and gender was also conducted. As shown in Table II, the results suggested that a higher expression of SLC22A4 $(\mathrm{OR}=5.219$, $\mathrm{P}=0.003)$, IL1R2 (OR=5.228, $\mathrm{P}=0.007), \mathrm{VNN} 3$ (OR=3.478, $\mathrm{P}=0.012)$ and female $(\mathrm{OR}=0.208, \mathrm{P}=0.005)$ might be independently associated with elevated NLR in CHF patients.

\section{Discussion}

The current study is the first to investigate the potential decisive factors associated with elevated NLR in CHF by microarray assay of genes, demonstrating that several genes including SLC22A4, IL1R2 and VNN3 may be independently associated with the elevation of NLR.

NLR reflecting the systemic inflammatory response has been consistently confirmed to affect the severity and prognosis of many cardiovascular diseases (6), which implies its role as a reliable prognostic index. NLR has also been found with similar independent prognostic power as NT-proBNP for older people with HF but with inexpensive and readily available testing properties (2). To be biologically reasonable, the capability of NLR by integrating two different yet complementary immune pathways may account for its predictive superiority of NLR. Neutrophils represent a subclinical inflammatory condition whereby endothelial damage and platelet aggregation are initiated by the release of pro-oxidants and prothrombotic substances (21). By contrast, reduced lymphocytes proportion represents a physiological stress and poor general health (22). Therefore, NLR actually acts as a balance of inflammation and stress response (5). Clinically, NLR has been found predictive for cardiac arrhythmias and mortality in patients with acute coronary syndromes. High NLR has also been found to be associated with arterial stiffness and high coronary calcium score. Moreover, elevated NLR has been repeatedly found a powerful predictor of worse clinical outcome with HF. Despite the significance of NLR as a prognostic index in the prognostication and risk stratification of cardiovascular disease including HF verified by many studies, NLR, like most inflammatory markers, is trapped in the lack of specificity to a certain disease. Therefore, several decisive factors associated with the elevation of NLR in CHF may aid in the prediction of prognosis of CHF in combination with NLR.

In the present study involving $197 \mathrm{CHF}$ patients, increased expressions of SLC22A4, IL1R2 and VNN3 were found in patients with elevated NLR and independent significant associations of SLC22A4, IL1R2 and VNN3 with elevated NLR in CHF patients were also revealed through a further comprehensive analysis including ROC, simple and multivariate regression analysis, which suggests the possible predictive role of SLC22A4, 

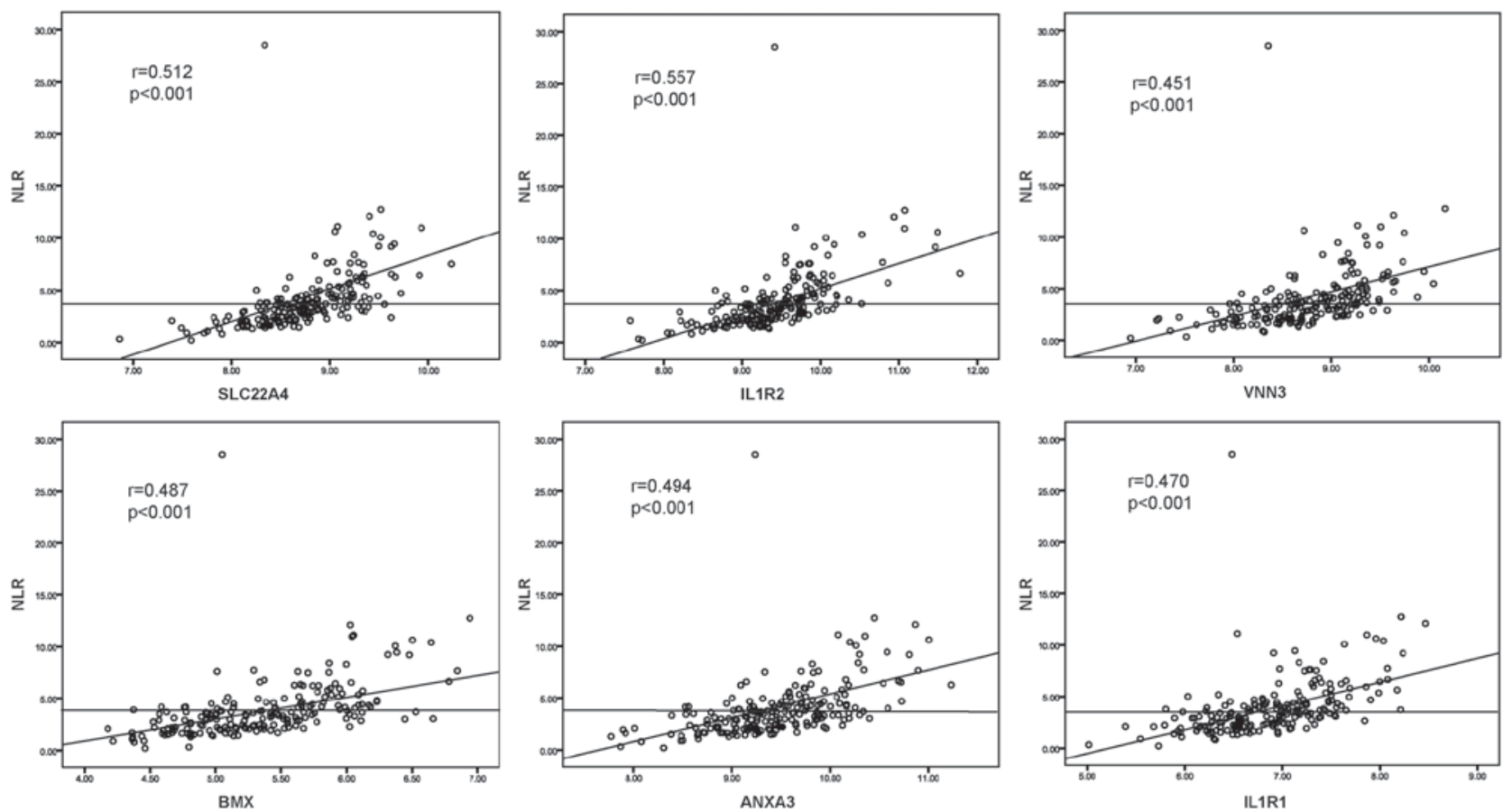

Figure 4. Pearson correlation analysis of the expression of the six selected genes with NLR. SLC22A4, solute carrier family 22 member 4; IL1R, interleukin-1 receptor; VNN3, vanin 3; BMX, BMX non-receptor tyrosine kinase; ANXA3, Annexin A3; NLR, meutrophil-to-lymphocyte ratio.

IL1R2 and VNN3 in NLR. Considering the significance of NLR as a prognostic index in $\mathrm{HF}$, the suggested genes as potential decisive factors for NLR may be hypothesized for the prediction of prognostication in CHF. Biologically, the detrimental effect of over-expressed SLC22A4 (also known as OCTN1) which acts as an organic cation transporter in cardiomyocytes has been demonstrated by a previous study (23). SLC22A4 was suggested to be a potential risk factor for torsade de pointes by intensifying quinidine-induced HERG block (23). In addition, OCTN1 was also found to be possibly associated with rheumatoid arthritis (24). Although modest, it is plausible to propose a deleterious effect of dysregulated SLC22A4 on CHF patients. Similarly, the inflammatory cytokine IL1R2 acting as an important component of IL1 signal pathway was found associated with several common cardiovascular diseases including coronary artery disease (25), hypertension (26), cardiac allograft rejection and coronary heart disease $(27,28)$. The inflammation-related function of IL1R2 may also support a role in the pathogenesis and prognosis of CHF. Moreover, the vanin family gene VNN3 is a secreted and membrane-bound ectoenzyme which converts pantetheine into pantothenic acid and cysteamine. A previous study screening a blood transcriptome-based molecular signature demonstrated that VNN3 may serve as a more specific and sensitive diagnostic biomarker for ST-segment-elevation myocardial infarction than traditional creatine kinase-MB or troponin (29). Additionally, the expression of VNN3 was also found to be upregulated in mesangial proliferative nephritis and skin psoriasis lesions, suggesting its proinflammatory activity in diseases $(30,31)$. Collectively, the findings of the present study may hint at the potential for SLC22A4, IL1R2 and VNN3 associated with altered NLR to predict the prognosis with CHF patients owing to their evidenced biological properties in cardiovascular and inflammatory diseases to some extent. However, their prognostic value remains undefined to date. Furthermore, the present study also indicated that female gender tended to present a lower NLR, potentially suggesting a favorable prognosis for female $\mathrm{CHF}$ patients, which was supported by a large-scale cohort study which demonstrated that women had a lower risk of death irrespective of cause of $\mathrm{HF}$ or left-ventricular ejection fraction (32).

To be noteworthy, the uniform cut-off of NLR remains undefined in the scientific community, so we used the mean 3.96 of NLR as the cut-off to define the elevation of NLR, while the cut-off utilized in several studies reporting a significant association of elevated NLR with a worse clinical outcome of CHF varied from 2.74 to $4.1(2,7,10,33)$. Therefore, it may indicate a comparatively high risk of mortality for $\mathrm{CHF}$ patients with NLR greater than 3.96 in this study. Accordingly, combined with the biological inherent property discussed previously, the genes SLC22A4, IL1R2 and VNN3 associated with the useful prognostic index NLR may be potential predictors of CHF prognosis in combination with NLR. However, further perspective studies are needed to confirm the findings.

Our study has certain limitations. First, the NLR cut-off based on mean, median or quantile was previously used widely to explore the diagnostic and prognostic role of NLR in most cardiovascular diseases due to the lack of a uniform cut-off of NLR defined by the scientific community, we therefore used the mean 3.96 of NLR as the cut-off to define the elevation of NLR, which may have resulted in misclassification of patients. Second, several well known pro-inflammatory and prognostic markers such as NT-proBNP, interleukin-6, high-sensitivity CRP and cardiac troponin were not analyzed and compared with the NLR due to the lack of available relevant information. Nevertheless, the present study also has unoverlooked redeeming feature. Although measurements of serum products were more common and convenient in the clinics, some genes with prognostic potential may be undetectable in serum. Moreover, it may suffer from limitations in providing a complete and unbiased genetic profile 
Table I. Results of logistic regression analysis adjusted by age and sex.

\begin{tabular}{|c|c|c|c|}
\hline \multirow[b]{2}{*}{ Variables } & \multicolumn{2}{|c|}{ Logistic regression } & \multirow[b]{2}{*}{ P-value } \\
\hline & OR & $95 \% \mathrm{CI}$ & \\
\hline \multicolumn{4}{|l|}{ SLC22A4 } \\
\hline Age, years ( $\geq 66$ vs. $<66)$ & 1.702 & $0.805-3.599$ & 0.164 \\
\hline Sex (female vs. male) & 0.311 & $0.122-0.789$ & $0.014^{\mathrm{a}}$ \\
\hline Expression (high vs. low) & 16.844 & $7.910-35.869$ & $<0.001^{\mathrm{c}}$ \\
\hline \multicolumn{4}{|l|}{ IL1R2 } \\
\hline Age, years ( $\geq 66$ vs. $<66)$ & 1.054 & $0.496-2.239$ & 0.891 \\
\hline Sex (female vs. male) & 0.252 & $0.100-0.636$ & $0.004^{\mathrm{b}}$ \\
\hline Expression (high vs. low) & 18.799 & $8.617-41.013$ & $<0.001^{\mathrm{c}}$ \\
\hline \multicolumn{4}{|l|}{ VNN3 } \\
\hline Age, years $(\geq 66$ vs. $<66)$ & 1.434 & $0.710-2.898$ & 0.315 \\
\hline Sex (female vs. male) & 0.294 & $0.120-0.720$ & $0.007^{\mathrm{b}}$ \\
\hline Expression (high vs. low) & 11.136 & $5.501-22.534$ & $<0.001^{\mathrm{c}}$ \\
\hline \multicolumn{4}{|l|}{ BMX } \\
\hline Age, years ( $\geq 66$ vs. $<66)$ & 1.076 & $0.526-2.202$ & 0.841 \\
\hline Sex (female vs. male) & 0.325 & $0.132-0.800$ & $0.014^{\mathrm{a}}$ \\
\hline Expression (high vs. low) & 11.716 & $5.742-23.908$ & $<0.001^{\mathrm{c}}$ \\
\hline \multicolumn{4}{|l|}{ ANXA3 } \\
\hline Age, years $(\geq 66$ vs. $<66)$ & 1.461 & $0.732-2.918$ & 0.282 \\
\hline Sex (female vs. male) & 0.390 & $0.163-0.931$ & $0.034^{\mathrm{a}}$ \\
\hline Expression (high vs. low) & 9.562 & $4.796-19.063$ & $<0.001^{\mathrm{c}}$ \\
\hline \multicolumn{4}{|l|}{ IL1R1 } \\
\hline Age, years ( $\geq 66$ vs. $<66)$ & 1.228 & $0.616-2.448$ & 0.559 \\
\hline Sex (female vs. male) & 0.263 & $0.108-0.636$ & $0.003^{\mathrm{b}}$ \\
\hline Expression (high vs. low) & 9.585 & $4.736-19.400$ & $<0.001^{\mathrm{c}}$ \\
\hline
\end{tabular}

${ }^{\mathrm{a}} \mathrm{P}<0.05 ;{ }^{\mathrm{b}} \mathrm{P}<0.01$; ${ }^{\mathrm{C}}<0.001$. SLC22A4, solute carrier family 22 member 4; IL1R2, interleukin-1 receptor 2; VNN3, vanin 3; BMX, BMX non-receptor tyrosine kinase; ANXA3, Annexin A3; OR, odds ratio; CI, confidence interval.

Table II. Result of multiple logistic regression analysis.

\begin{tabular}{llll}
\hline & \multicolumn{2}{c}{ Logistic regression } \\
\cline { 2 - 3 } Variables & OR & \multicolumn{2}{c}{$95 \%$ CI } \\
\hline Age, years ( $\geq 66$ vs. <66) & 1.278 & $0.532-3.071$ & 0.584 \\
Gender (female vs. male) & 0.208 & $0.069-0.629$ & $0.005^{\text {b }}$ \\
SLC22A4 (high vs. low expression) & 5.219 & $1.762-15.458$ & $0.003^{\text {b }}$ \\
IL1R2 (high vs. low expression) & 5.228 & $1.560-17.515$ & $0.007^{\text {b }}$ \\
VNN3 (high vs. low expression) & 3.478 & $1.322-9.195$ & $0.012^{\text {a }}$ \\
BMX (high vs. low expression) & 2.012 & $0.775-5.221$ & 0.151 \\
ANXA3 (high vs. low expression) & 1.870 & $0.180-2.173$ & 0.225 \\
IL1R1 (high vs. low expression) & 0.625 & 0.460 \\
\hline
\end{tabular}

${ }^{\mathrm{a}} \mathrm{P}<0.05 ;{ }^{\mathrm{b}} \mathrm{P}<0.01$. SLC22A4, solute carrier family 22 member 4; IL1R2, interleukin-1 receptor 2; VNN3, vanin 3; BMX, BMX non-receptor tyrosine kinase; ANXA3, Annexin A3; OR, odds ratio; CI, confidence interval.

of the circulating peripheral blood compartment of an individual, which may be affected by factors such as preprocessing of samples, cellular contamination. Collectively, the detection of gene expression from peripheral blood may be a significant 
supplement to routine measurement of serum products when necessary in the clinic.

In summary, the current study was designed to identify DEGs associated with elevated NLR, which may be candidate predictors for CHF prognosis. A total of 31 DEGs were revealed, of which three potential decisive factors SLC22A4, IL1R2 and VNN3 independently associated with elevated NLR were focused. Nevertheless, the present study was devised using a bioinformatics strategy, further clinical studies are warranted to verify the findings.

\section{Acknowledgements}

Not applicable.

\section{Funding}

No funding was received.

\section{Availability of data and materials}

All data generated or analyzed during this study are included in this published article.

\section{Authors' contributions}

GXW conceived and designed the study, and drafted the manuscript. LHJ, WBX and LC performed the statistical analysis, data collation and interpretation. YGZ designed the study, and reviewed and edited the manuscript. All authors have read and approved the final manuscript.

\section{Ethics approval and consent to participate}

Not applicable.

\section{Consent for publication}

Not applicable.

\section{Competing interests}

The authors declare that they have no competing interests.

\section{References}

1. Tasal A, Erturk M, Uyarel H, Karakurt H, Bacaksiz A, Vatankulu MA, Turfan M, Sonmez O, Erdogan E and Ergelen M: Utility of the neutrophil to lymphocyte ratio for predicting in-hospital mortality after levosimendan infusion in patients with acute decompensated heart failure. J Cardiol 63: 418-423, 2014.

2. Yan W, Li RJ, Jia Q, Mu Y, Liu CL and He KL: Neutrophil-to-lymphocyte ratio compared to N-terminal pro-brain natriuretic peptide as a prognostic marker of adverse events in elderly patients with chronic heart failure. J Geriatr Cardiol 14: 127-134, 2017.

3. Ostrowska M, Ostrowski A, Łuczak M, Jaguszewski M, Adamski P, Bellwon J, Rynkiewicz A and Gruchała M: Basic laboratory parameters as predictors of in-hospital death in patients with acute decompensated heart failure: Data from a large single-centre cohort. Kardiol Pol 75: 157-163, 2017.

4. Ho KK, Anderson KM, Kannel WB, Grossman W and Levy D: Survival after the onset of congestive heart failure in Framingham Heart Study subjects. Circulation 88: 107-115, 1993.
5. Afari ME and Bhat T: Neutrophil to lymphocyte ratio (NLR) and cardiovascular diseases: An update. Expert Rev Cardiovasc Ther 14: 573-577, 2016.

6. Bhat T, Teli S, Rijal J, Bhat H, Raza M, Khoueiry G, Meghani M, Akhtar $M$ and Costantino T: Neutrophil to lymphocyte ratio and cardiovascular diseases: A review. Expert Rev Cardiovasc Ther 11: 55-59, 2013.

7. Wasilewski J, Pyka Ł, Hawranek M, Osadnik T, Kurek A, Skrzypek M, Niedziela J, Desperak P, Kułaczkowska Z, Brzezina M, et al: Prognostic value of neutrophil-to-lymphocyte ratio in predicting long-term mortality in patients with ischemic and nonischemic heart failure. Pol Arch Med Wewn 126: 166-173, 2016.

8. Uthamalingam S, Patvardhan EA, Subramanian S, Ahmed W, Martin W, Daley M and Capodilupo R: Utility of the neutrophil to lymphocyte ratio in predicting long-term outcomes in acute decompensated heart failure. Am J Cardiol 107: 433-438, 2011.

9. Benites-Zapata VA, Hernandez AV, Nagarajan V, Cauthen CA, Starling RC and Tang WH: Usefulness of neutrophil-to-lymphocyte ratio in risk stratification of patients with advanced heart failure. Am J Cardiol 115: 57-61, 2015.

10. Yan W, Liu C, Li R, Mu Y, Jia Q and He K: Usefulness of the neutrophil-to-lymphocyte ratio in predicting adverse events in elderly patients with chronic heart failure. Int Heart J 57: 615-621, 2016.

11. Mei Z, Shi L, Wang B, Yang J, Xiao Z, Du P, Wang Q and Yang W: Prognostic role of pretreatment blood neutrophil-to-lymphocyte ratio in advanced cancer survivors: A systematic review and meta-analysis of 66 cohort studies. Cancer Treat Rev 58: 1-13, 2017.

12. Shannon CP, Balshaw R, Chen V, Hollander Z, Toma M, McManus BM, Fitzgerald JM, Sin DD, Ng RT and Tebbutt SJ: A novel approach to identifying marker genes and estimating the cellular composition of whole blood from gene expression profiles. bioRxiv 2: 38794, 2016

13. Irizarry RA, Hobbs B, Collin F, Beazer-Barclay YD, Antonellis KJ, Scherf U and Speed TP: Exploration, normalization, and summaries of high density oligonucleotide array probe level data. Biostatistics 4: 249-264, 2003.

14. Troyanskaya O, Cantor M, Sherlock G, Brown P, Hastie T, Tibshirani R, Botstein D and Altman RB: Missing value estimation methods for DNA microarrays. Bioinformatics 17: 520-525, 2001.

15. Ritchie ME, Phipson B, Wu D, Hu Y, Law CW, Shi W and Smyth GK: Limma powers differential expression analyses for RNA-sequencing and microarray studies. Nucleic Acids Res 43: e47, 2015 .

16. Szklarczyk D, Franceschini A, Wyder S, Forslund K, Heller D, Huerta-Cepas J, Simonovic M, Roth A, Santos A, Tsafou KP, et al: STRING v10: Protein-protein interaction networks, integrated over the tree of life. Nucleic Acids Res 43 (Database Issue): D447-D452, 2015.

17. Gene Ontology Consortium: The Gene Ontology (GO) project in 2006. Nucleic Acids Res 34 (Database Issue): D322-D326, 2006.

18. Kanehisa M and Goto S: KEGG: Kyoto encyclopedia of genes and genomes. Nucleic Acids Res 28: 27-30, 2000.

19. Yu G, Wang LG, Han Y and He QY: clusterProfiler: An R package for comparing biological themes among gene clusters. OMICS 16: 284-287, 2012.

20. Budczies J, Klauschen F, Sinn BV, Győrffy B, Schmitt WD, Darb-Esfahani S and Denkert C: Cutoff Finder: A comprehensive and straightforward Web application enabling rapid biomarker cutoff optimization. PLoS One 7: e51862, 2012.

21. Weber C, Zernecke A and Libby P: The multifaceted contributions of leukocyte subsets to atherosclerosis: Lessons from mouse models. Nat Rev Immunol 8: 802-815, 2008.

22. Azab B, Zaher M, Weiserbs KF, Torbey E, Lacossiere K, Gaddam S, Gobunsuy R, Jadonath S, Baldari D, McCord D and Lafferty $\mathrm{J}$ : Usefulness of neutrophil to lymphocyte ratio in predicting short- and long-term mortality after non-ST-elevation myocardial infarction. Am J Cardiol 106: 470-476, 2010.

23. McBride BF, Yang T, Liu K, Urban TJ, Giacomini KM, Kim RB and Roden DM: The organic cation transporter, OCTN1, expressed in the human heart, potentiates antagonism of the HERG potassium channel. J Cardiovasc Pharmacol 54: 63-71, 2009.

24. Taubert D, Lazar A, Grimberg G, Jung N, Rubbert A, Delank KS, Perniok A, Erdmann E and Schömig E: Association of rheumatoid arthritis with ergothioneine levels in red blood cells: A case control study. J Rheumatol 33: 2139-2145, 2006. 
25. Long F, Wang L, Yang L, Ji Z and Hu Y: Screening hub genes in coronary artery disease based on integrated analysis. Cardiol J: Oct 5, 2017 (Epub ahead of print).

26. Stoynev N, Dimova I, Rukova B, Hadjidekova S, Nikolova D, Toncheva D and Tankova T: Gene expression in peripheral blood of patients with hypertension and patients with type 2 diabetes. J Cardiovasc Med (Hagerstown) 15: 702-709, 2014.

27. Mehra MR, Uber PA and Benitez RM: Gene-based bio-signature patterns and cardiac allograft rejection. Heart Fail Clin 6: 87-92, 2010.

28. Chen X, Chen X, Xu Y, Yang W, Wu N, Ye H, Yang JY, Hong Q, Xin Y, Yang MQ, et al: Association of six CpG-SNPs in the inflammation-related genes with coronary heart disease. Hum Genomics 10 (Suppl 2): S21, 2016.

29. Park HJ, Noh JH, Eun JW, Koh YS, Seo SM, Park WS, Lee JY, Chang K, Seung KB, Kim PJ and Nam SW: Assessment and diagnostic relevance of novel serum biomarkers for early decision of ST-elevation myocardial infarction. Oncotarget 6: 12970-12983, 2015.

30. Lu Y, Chen X, Yin Z, Zhu S, Wu D and Chen X: Screening for potential serum biomarkers in rat mesangial proliferative nephritis. Proteomics 16: 1015-1022, 2016.
31. JansenPA,KamsteegM,Rodijk-OlthuisD,van Vlijmen-WillemsIM, de Jongh GJ, Bergers M, Tjabringa GS, Zeeuwen PL and Schalkwijk J: Expression of the vanin gene family in normal and inflamed human skin: Induction by proinflammatory cytokines. J Invest Dermatol 129: 2167-2174, 2009.

32. O'Meara E, Clayton T, McEntegart MB, McMurray JJ, Piña IL, Granger CB, Ostergren J, Michelson EL, Solomon SD, Pocock S, et al: Sex differences in clinical characteristics and prognosis in a broad spectrum of patients with heart failure: Results of the Candesartan in Heart failure: Assessment of Reduction in Mortality and morbidity (CHARM) program. Circulation 115: 3111-3120, 2007

33. Cakici M, Cetin M, Doğan A, Oylumlu M, Aktürk E, Polat M, Suner A and Abuş S: Neutrophil to lymphocyte ratio predicts poor functional capacity in patients with heart failure. Turk Kardiyol Dern Ars 42: 612-620, 2014.

(i) (9) This work is licensed under a Creative Commons

EY No Attribution-NonCommercial-NoDerivatives 4.0 International (CC BY-NC-ND 4.0) License. 